\title{
In an in vitro model of human tuberculosis, monocyte-microglial networks regulate matrix metalloproteinase- 1 and -3 gene expression and secretion via a p38 mitogen activated protein kinase-dependent pathway
}

Justin A Green'1, Lucinda Rand', Rachel Moores', Shruti Dholakia', Theodore Pezas', Paul T Elkington ${ }^{1,2}$ and Jon S Friedland ${ }^{1 *}$

\begin{abstract}
Background: Tuberculosis (TB) of the central nervous system (CNS) is characterized by extensive tissue inflammation, driven by molecules that cleave extracellular matrix such as matrix metalloproteinase (MMP)-1 and MMP-3. However, relatively little is known about the regulation of these MMPs in the CNS.

Methods: Using a cellular model of CNS TB, we stimulated a human microglial cell line (CHME3) with conditioned medium from Mycobacterium tuberculosis-infected primary human monocytes (CoMTb). MMP-1 and MMP-3 secretion was detected using ELISAs confirmed with casein zymography or western blotting. Key results of a phospho-array profile that detects a wide range of kinase activity were confirmed with phospho-Western blotting. Chemical inhibition (SB203580) of microglial cells allowed investigation of expression and secretion of MMP-1 and MMP-3. Finally we used promoter reporter assays employing full length and MMP-3 promoter deletion constructs. Student's t-test was used for comparison of continuous variables and multiple intervention experiments were compared by one-way ANOVA with Tukey's correction for multiple pairwise comparisons.

Results: CoMTb up-regulated microglial MMP-1 and MMP-3 secretion in a dose- and time-dependent manner. The phospho-array profiling showed that the major increase in kinase activity due to CoMTb stimulation was in p38 mitogen activated protein kinase (MAPK), principally the $a$ and $y$ subunits. p38 phosphorylation was detected at 15 minutes, with a second peak of activity at 120 minutes. High basal extracellular signal-regulated kinase activity was further increased by CoMTb. Secretion and expression of MMP-1 and MMP-3 were both p38 dependent. CoMTb stimulation of full length and MMP-3 promoter deletion constructs demonstrated up-regulation of activity in the wild type but a suppression site between -2183 and -1612 bp.
\end{abstract}

Conclusions: Monocyte-microglial network-dependent MMP-1 and MMP-3 gene expression and secretion are dependent upon p38 MAPK in tuberculosis. p38 is therefore a potential target for adjuvant therapy in CNS TB.

Keywords: Tuberculosis, Central nervous system, Matrix metalloproteinase, Immunopathology, Mitogen-activated protein kinase

\footnotetext{
* Correspondence: j.friedland@imperial.ac.uk

${ }^{1}$ Section of Infectious Diseases and Immunity and the Imperial College

Wellcome Trust Centre for Clinical Tropical Medicine, Hammersmith Campus,

Imperial College London, London W12 ONN, UK

Full list of author information is available at the end of the article
} 


\section{Background}

Tuberculosis (TB) remains a deadly disease responsible for approximately 1.5 million deaths a year, many caused by disseminated disease involving the central nervous system (CNS) [1]. During active disease, infection of monocytic cells by Mycobacterium tuberculosis (M.tb) results in an intense host-driven inflammatory response that contributes not only to pathogen death, but also to local tissue destruction and immunopathology. In CNS $\mathrm{TB}$, this inflammatory reaction is not well-tolerated and is responsible for infarction, hydrocephalus, neuronal damage and death.

Monocytic cell-derived matrix metalloproteinases ( $M$ MPs) are central mediators of TB-driven pulmonary tissue destruction due to their ability to degrade fibrillar collagens [2]. Indeed this family of 23 proteases with differing, but overlapping, substrates can degrade all elements of the extracellular matrix at neutral $\mathrm{pH}$, differentiating them from other proteases that might also be induced in inflammatory situations [3,4]. MMPs perform multiple roles in development, reproduction and the normal immune response, such as facilitating leucocyte recruitment, processing cytokines and chemokines, activating defensins and remodelling matrix [5]. In the CNS, MMPs are known to have a number of substrates, such as dystroglycan, a transmembrane receptor that anchors astrocyte endfeet to the basement membrane via laminin binding [6]. Cleavage of these molecules contributes to blood-brain barrier breakdown. The resident CNS innate immune cell is the microglia, most likely of mesodermal monocyte-lineage origin [7]. We and others have previously shown that microglia secrete high concentrations of MMPs in CNS TB [8-10]. MMP-1, MMP-3, MMP-8 and MMP-9 are found at high concentrations in the cerebral spinal fluid (CSF) of CNS TB patients [11]. In addition, MMP-1, MMP-3 and -9, but not the specific tissue inhibitor of metalloproteinase (TIMP) -1, are present in CNS tuberculomas [9,12]. Specifically MMP-1 is an interstitial collagenase able to digest aggrecan and collagen at neutral $\mathrm{pH}$, and MMP-3 is a stromelysin with substrates such as fibronectin, laminin and type IV collagen [3]. Finally dexamethasone, a specific and proven adjuvant to anti-tuberculous chemotherapy that improves patient outcomes, specifically suppresses MMPs, but not TIMPs, in CNS TB patients [11,13].

Intracellular signalling is critical to control of MMP transcription and secretion. We have shown that nuclear factor- $\mathrm{kB}(\mathrm{NF}-\mathrm{kB})$ and activator protein (AP)-1 pathways are activated both in vitro and in patients with $\mathrm{CNS}$ TB [9]. Furthermore, the bacteriostatic TB drug para-aminosalicyclic acid exerts its anti-TB activity in part due to inhibition of the prostaglandin pathway, thereby reducing MMP-1 secretion and tissue destruction [14]. Upstream of these signalling pathways are the mitogen-activated protein kinases (MAPKs). The three main pathways are p38, c-Jun N-terminal kinase (JNK) and extracellular signal-regulated kinase (ERK)-1/2. The extracellular signal-regulated MAPK are involved in cell turnover and phosphorylation of transcription factors and microtubuleassociated proteins, whereas the p38 MAP kinase subfamily regulates cell differentiation, apoptosis and cellular response to inflammation. p38 MAPK may be an important point of divergence in the regulation of MMP and TIMP secretion in pulmonary TB [15]. In the CNS, MMP-9 secretion by astrocytes was dependent upon an IL- $1 \beta / M A P$ kinase/AP-1 pathway [16].

In this study, we tested the hypothesis that MAPK pathways are key factors in the control of microglial MMP gene expression and secretion using a cellular model of CNS TB. We present evidence that the $\mathrm{p} 38$ pathway is the principle regulator of MMP secretion in microglia, causing divergent secretion of MMP-1 and MMP-3 and their associated TIMPs.

\section{Methods \\ Reagents}

Chemicals were from Sigma-Aldrich (Gillingham, UK), tissue culture materials from Invitrogen (Paisley, UK) and tissue culture plastic from TPP (Trasadingen, Switzerland) unless otherwise stated. Inhibition experiments were performed with a two-hour pre-incubation of the specific p38 chemical inhibitor SB203580 from Promega (Southampton, UK) at concentrations as shown.

\section{M.tb culture}

The virulent M.tb strain H73Rv Pasteur was cultured in Middlebrook 7H9 broth (BD Biosciences, Oxford, UK) with $10 \%$ OADC enrichment medium, $0.2 \%$ glycerol and $0.02 \%$ Tween 80 with agitation. M.tb at optical density (OD) 0.6 (Biowave Cell Density Meter; WPA, Cambridge, $\mathrm{UK}$ ), corresponding to $10^{8}$ colony forming units $/ \mathrm{ml}$ (cfu/ $\mathrm{ml}$ ) was used for infection. Endotoxin level was $<0.03 \mathrm{ng} /$ $\mathrm{ml}$ lipopolysaccharide (LPS) (Amoebocyte lysate assay, Associates of Cape Cod, Falmouth, MA, USA).

\section{Cell culture and M.tb infection}

Monocytes were isolated from healthy blood donors (UK Blood Transfusion Service) by Ficoll-Paque (GE Healthcare, Little Chalfont, UK) density gradient centrifugation and adherence purification [17]. Monocytes were infected with $M . t b$ at a multiplicity of infection of 1 . At 24 hours, cell culture medium was collected and $0.2 \mu \mathrm{m}$ was sterilefiltered (Nalgene, Hereford, UK) to remove infectious particles but not MMPs [18]. Conditioned medium from $M$. $t b$-infected monocytes was termed CoMTb and control medium from uninfected monocytes was CoMCont.

Human CHME3 microglial cells (used with permission of Marc Tardieu, Paris, France and kindly provided by 
Nicola Woodroofe, Sheffield Hallam University, UK) were maintained in DMEM with 10\% FCS (Biosera, Ringmer, $\mathrm{UK}$ ) and $3 \mathrm{mM}$ glutamine. Cells were seeded at 35,000 to $50,000 \mathrm{cells} / \mathrm{cm}^{2}$ for experiments, which were performed in macrophage serum-free medium (MSFM).

\section{RNA extraction, CDNA synthesis and quantitative reverse- transcribed PCR (qRT-PCR)}

Microglia were lysed with TRI reagent and total RNA was extracted. cDNA was prepared from 1 ug RNA and reverser transcribed using $2 \mu \mathrm{l}$ of random primers and 200 units SuperScript III reverse transcriptase (both Invitrogen), according to supplier's instructions. Quantitative PCR (qPCR) was performed as previously described [9]. The cycle threshold $(\mathrm{Ct})$ at which amplification entered the exponential phase was determined and this number was used to indicate the amount of target RNA in each sample. MMP Cts were normalized to ribosomal 18S mRNA.

\section{Casein zymography}

We loaded $20 \mu \mathrm{l}$ of cell culture medium with $5 \mu \mathrm{l}$ of loading buffer $(0.25 \mathrm{M}$ Tris $\mathrm{pH} 6.8,50 \%$ glycerol, 5\% SDS and bromophenol blue crystals) on $12 \%$ casein gels (Invitrogen). Gels were run at $125 \mathrm{~V}$ for 2.5 hours (buffer $25 \mathrm{mM}$ Tris, $190 \mathrm{mM}$ glycine and 0.1\% SDS). After one hour incubation in $2.5 \%$ Triton-X, gels were transferred to collagenase buffer ( $55 \mathrm{mM}$ Tris base, $200 \mathrm{mM} \mathrm{NaCl}, 5$ $\mathrm{mM} \mathrm{CaCl} 2$ and $0.02 \%$ Brij; $\mathrm{pH}$ 7.6) for 40 hours at $37^{\circ} \mathrm{C}$. Gels were stained with $0.2 \%$ Coomassie blue (Pharmacia Biotech, Sweden) for one hour before de-staining in acetic $\mathrm{acid} / \mathrm{methanol} /$ water (1:3:6), and $5 \mathrm{ng}$ of MMP-1 standard (Merck, Germany) was run on each gel. Gels were analyzed densitometrically by Scion Image Analysis (NIH Image version 1.61, Bethesda, MD, USA) normalized to standard.

\section{Western blotting}

Standard MMP-1 western blotting was performed as described [19]. Overnight incubation in 1:1,000 MMP-1 primary antibody (The Binding Site, Birmingham, UK) in 5\% milk protein was followed by washing and incubation with 1:2000 peroxidase-conjugate secondary antibody for an hour (The Binding Site). Bands were visualized by chemiluminescence with the ECL Plus system (GE Healthcare) according to manufacturer's instructions. MMP-3 western blotting was performed using 1:5,000 rabbit anti-MMP-3 primary antibody (Chemicon, Millipore, Watford, UK) in $5 \%$ bovine serum albumin and 1:2,000 horseradish peroxidase (HRP)-linked anti-rabbit secondary (Cell Signalling Technology, Danvers, MA, US) in 5\% milk protein. For MAPK experiments, cells were lysed using $200 \mu \mathrm{l}$ of SDS sample buffer (62.5 mM Tris pH 6.8, 2\% SDS, 10\% glycerol, $50 \mathrm{mM}$ DTT and $0.01 \%$ bromophenol blue) and immediately frozen at $-80^{\circ} \mathrm{C}$. Then, $40 \mu \mathrm{l}$ aliquots were run on western gels as described and probed with 1:1,000 rabbit primary antibodies to both phosphorylated and unphosphorylated forms of p38 or ERK MAPK. 1:2,000 dilution of anti-rabbit HRP-linked secondary antibody was used (all Cell Signalling Technology).

\section{Luminex analysis}

MMP-1 and MMP-3 concentrations were analyzed by Fluorokine MAP profiling kit according to the manufacturer's protocol (R\&D systems) on the Luminex platform (Biorad, Hemel Hempstead, UK). The minimum level of detection for MMP-1 was $10 \mathrm{pg} / \mathrm{ml}$ and $15 \mathrm{pg} / \mathrm{ml}$ for MMP-3.

\section{MMP-3, TIMP-1 and -2 ELISAs}

MMP-3 and TIMP-1 and TIMP-2 concentrations in cell culture medium were measured by ELISA (R\&D Systems) according to the manufacturer's instructions. The lower limit of detection was $30 \mathrm{pg} / \mathrm{ml}$.

\section{Phospho-MAPK array}

The Proteome Profiler phospho-array (R\&D systems) was used to investigate multiple kinase pathways. We stimulated $10^{6}$ microglia with CoMCont or CoMTb for $30 \mathrm{mi}-$ nutes. The phospho-array was performed according to the manufacturer's protocol and developed with the ECL system (GE Healthcare). Densitometric analysis of each array was performed using NIH Image version 1.61. The Proteome Profiler kit measured relative MAPK and related serine/threonine kinase phosphorylation status. Six positive controls are incorporated onto the membrane. Mean intensity was calculated for CoMCont and CoMTb. Data are presented as the difference between these mean intensities.

\section{Promoter reporter analysis}

Microglial cells were co-transfected with MMP-3 promoter constructs and a control reporter plasmid, constitutively expressing Renilla luciferase, with Fugene HD (Roche). Cells were stimulated after 18 hours, and luciferase activity analyzed 24 hours later by the Dual-Luciferase Reporter Assay System (Promega) on a luminometer (Medical Devices, Wokingham, UK). Renilla luciferase activity was used to normalize firefly luciferase activity to control for transfection efficiency.

\section{Statistics}

Data are presented as means $( \pm$ SD) of three samples and represent experiments performed in triplicate on at least two occasions, unless otherwise stated. Paired groups were compared with Student's $t$-test. Multiple intervention experiments were compared by one-way analysis of variance (ANOVA) and Tukey's correction for multiple pairwise comparisons with equal variance tested by Levene's test. $P<0.05$ was taken as significant. All analysis was done using SPSS software (version 15.0, SPSS corp., Chicago, IL, USA). 


\section{Results}

Monocyte-microglial networks drive MMP-1 and MMP-3 secretion from human microglia

Increasing concentrations of CoMTb but not CoMCont resulted in greater secretion of MMP-1 and MMP-3 in a dose-dependent manner (Figure 1A and B). Specifically, MMP-1 secretion showed a stepwise increase in secretion 9.2-fold higher at the 1:10 dilution of CoMTb compared to CoMCont and reaching 27.9-fold greater at 1:2 dilutions (all $P<0.01$ ). MMP-3 secretion was 12 -fold higher at the 1:10 dilution of CoMTb than the equivalent CoMCont concentration and 58-fold at the $1: 2$ dilution $(P<0.01)$. Since there was no further increase in MMP-3 secretion after a 1:5 dilution of CoMTb, this concentration was subsequently used to stimulate cells in all experiments.
MMP-1 and MMP-3 kinetic experiments demonstrated a significant difference in MMP secretion in response to CoMTb compared to CoMCont (Figure $1 \mathrm{C}$ and $\mathrm{D}$ ). MMP-1 activity was detectable at 24 hours and maximal at 96 hours with a 5.3-fold change from CoMCont $(P<0.01)$. MMP-3 secretion peaked at 72 hours with a 58 fold increase, but there was no further increase at 96 hours $(P<0.01)$. Therefore, cell culture supernatants were harvested at 72 hours in subsequent experiments.

\section{Phospho-array analysis of CoMTb-stimulated microglia}

Next, we investigated the intracellular signalling pathways regulating MMP-1/3 secretion using phosphor-array screening. The ERK and AKT pathways were activated in CoMCont-stimulated cells. At 30 minutes, CoMTb-
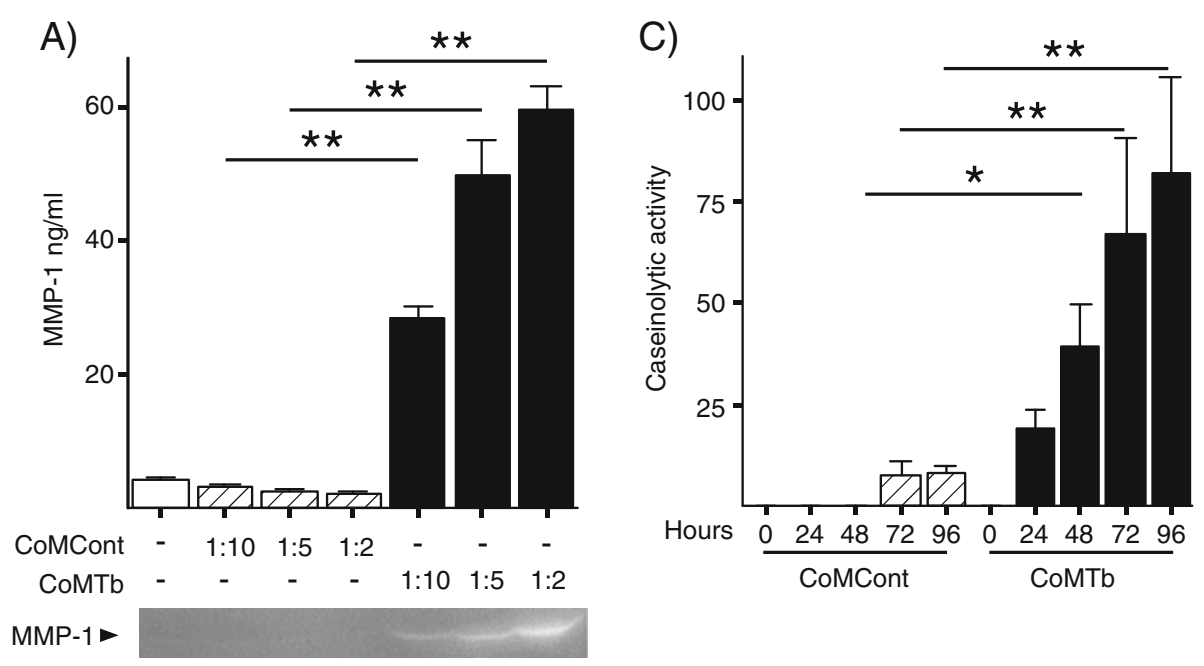

B)

D)
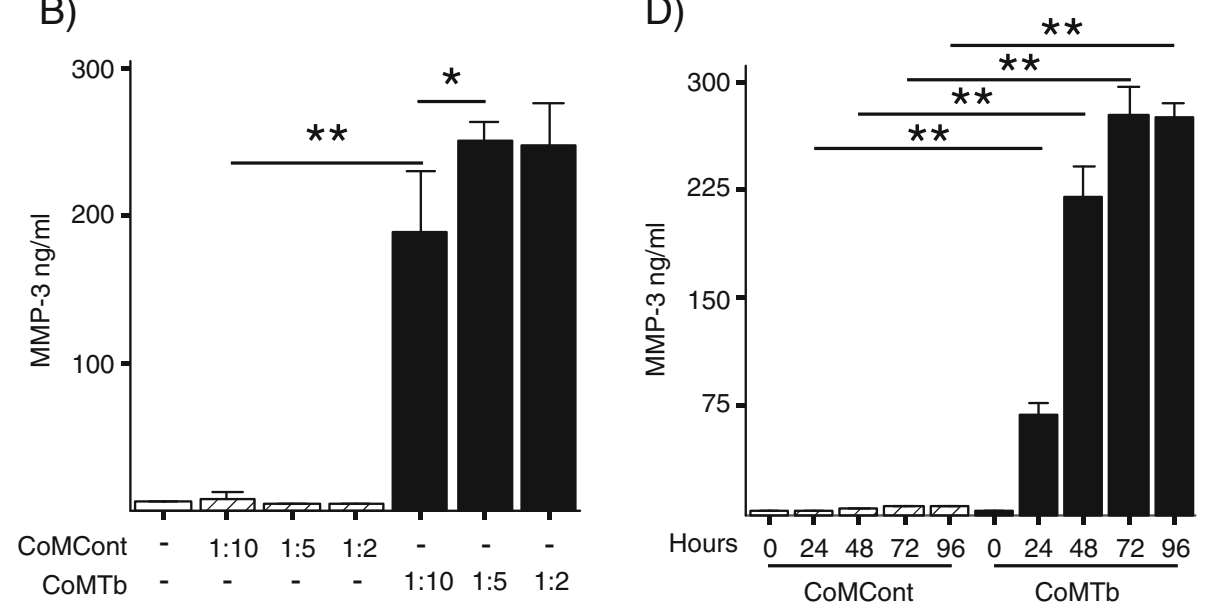

Figure 1 Matix metalloproteinsase (MMP)-1 and MMP-3 secretion is up-regulated by conditioned media from tuberculosis (TB)infected monocytes (CoMTb) in a dose- and time-dependent fashion. (A and B) Microglial cells were stimulated with control medium (open bars), or increasing concentrations of conditioned media from control monocytes (CoMCont) (diagonal hatched bars) and CoMTb (solid bars); 72hour supernatants were analyzed by Luminex for MMP-1 or MMP-3. A representative casein zymogram is also shown (A). Kinetics of MMP-1 activity (C) and MMP-3 secretion (D) demonstrate progressive increase in concentration for up to 72 hours. Bars represent mean values \pm SD of three samples, representative of at least duplicate experiments performed in triplicate. ${ }^{*} P<0.05,{ }^{* *} \mathrm{P}<0.01$. 
A)

\section{CoMCont}

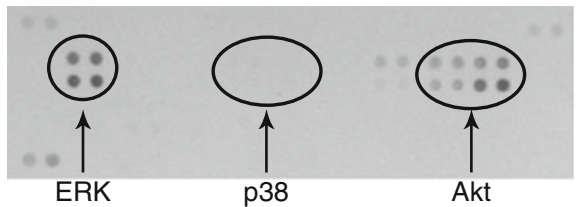

\section{CoMTb}

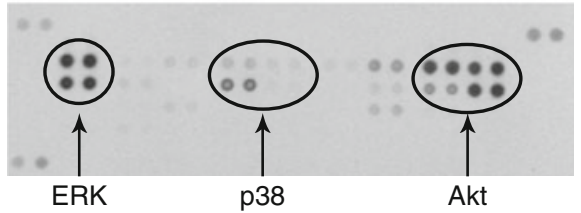

B)

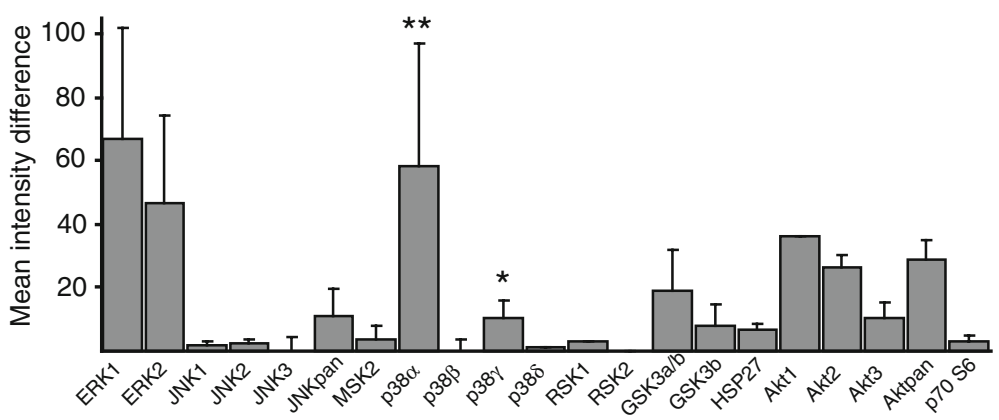

C)

CoMCont CoMTb

mins $0 \quad 1530601200153060120$

phospho-p38

total-p38 $-m-7-m-\cdots+\cdots$

D)

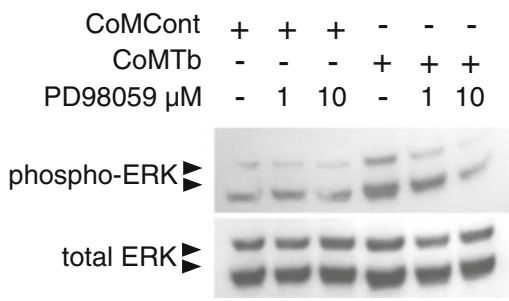

Figure 2 Conditioned media from tuberculosis (TB)-infected monocytes (CoMTb) drive phosphorylation of the extracellular signalregulated kinase (ERK), p38 and AKT mitogen-activated protein kinase (MAPK) pathways in microglial cells. (A) Proteome profiler array of conditioned media from control monocytes (CoMCont)- and CoMTb-stimulated microglia at 30 minutes. CoMTb drives p38 activity and further phosphorylation of constitutive ERK 1/2 and AKT activity. (B) Densitometric analysis of phospho-array. Densitometric analysis of differences in mean spot intensity between CoMCont- and CoMTb-stimulated cells is presented, demonstrating that the p38a and p38y subunits are the principal CoMTb up-regulated MAP kinases. (C) p38 phosphorylation kinetics. CoMTb drives up-regulation of p38 at 15 minutes, with a second peak at 120 minutes. (D) CoMCont-stimulated microglial ERK activity at 30 minutes shows high basal activity further up-regulated by CoMTb. Only CoMTb activity was blocked by the inhibitor PD98059. Representative data from three independent experiments are shown. Bars represent mean $+/$ - standard error of the mean from three independent experiments. ${ }^{*} P<0.05,{ }^{* *} \mathrm{P}<0.01$. 


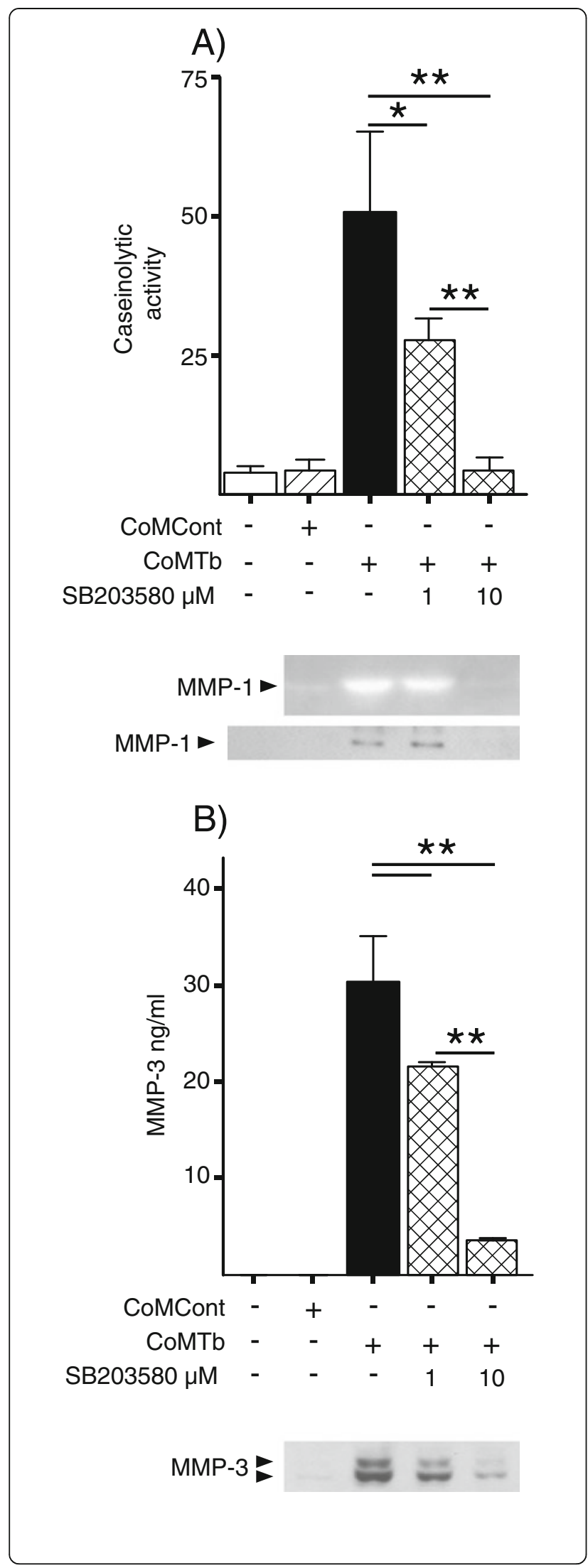

Figure 3 p38 phosphorylation controls both matix metalloproteinsase (MMP)- 1 and MMP-3 secretion in conditioned media from tuberculosis (TB)-infected monocytes (CoMTb)-stimulated microglia. Microglial cells were incubated with SB203580 for 2 hours, and then stimulated with CoMTb. (A) MMP-1 activation and secretion was analyzed at 72 hours by casein zymography (representative gel shown) and western blotting. (B) MMP-3 activity was analyzed by ELISA and western blotting. p38 inhibition suppressed CoMTb MMP-1 and MMP-3 suppression in a dose-dependent manner. Bars represent mean values \pm SD of three samples, representative of at least duplicate experiments performed in triplicate. Data were analyzed by one-way analysis of variance, followed by Tukey's multiple comparison test. ${ }^{*} P<0.05$, ${ }^{* *} P<0.01$.

stimulated multiple signalling pathways including ERK, p38, AKT, GSK, JNK and HSP-27 (Figure 2A). Densitometric analysis of differences in mean spot intensity between stimulated and unstimulated cells demonstrated that ERK-1/2 and AKT phosphorylation were up-regulated to the greatest extent in CoMTb-stimulated cells (Figure 2B). The increases in different p38 isoforms were divergent. CoMTb stimulated p38 $\alpha$ phosphorylation $311 \%$ more than CoMCont $(P<0.05)$ and $\mathrm{p} 38 \gamma$ phosphorylation increased by $154 \%(P<0.05)$. $\mathrm{p} 38 \beta$ and $\mathrm{p} 38 \delta$ phosphorylation did not change.

To investigate phospho-array findings further, kinetic Western blot analysis of CoM-stimulated p38 phosphorylation in microglia was performed over 120 minutes (Figure 2C). Minimal basal phosphorylation was seen. CoMTb stimulated p38 phosphorylation at 15 minutes, which waned by 60 minutes. However, a second peak of phosphorylation was detected at 2 hours. At 30 minutes, basal ERK phosphorylation was detected. Although CoMTb up-regulated ERK phosphorylation was blocked by PD98059 in a dose-dependent manner, the basal phosphorylation made subsequent interpretation of the effect of ERK hard to interpret, and so it was not studied further (Figure 2D).

\section{Regulation of MMP-1 and MMP-3 secretion by $\mathrm{p} 38$}

To dissect the role of the p38 pathway in control of MMP-1 and MMP-3 secretion, a specific chemical inhibitor (SB203580) was pre-incubated with microglia prior to CoMTb stimulation. MMP-1 secretion was suppressed by $45.6 \%$ by $1 \mu \mathrm{M}$ and $92 \%$ by $10 \mu \mathrm{M}$ SB $203580(P<0.05$ and $P<0.01$ respectively) (Figure 3A). MMP-3 was also p38-dependent; 1 and $10 \mu \mathrm{M}$ SB203580 reduced MMP-3 secretion by $28.8 \%$ and $83.2 \%$ respectively (both $P<0.01$ ) (Figure 3B).

MMP-1 and MMP-3 gene expression were investigated by qRT-PCR. MMP-1 gene expression was up-regulated by CoMTb (Figure 4A). This up-regulation was suppressed by inhibition of the p38 MAPK. MMP-3 mRNA accumulation was also decreased by p38 inhibition (Figure 4B), consistent with secretion data. 
The MMP-3 promoter is up-regulated by CoMTb and has a suppression site between -2183 and -1612 bp

The MMP-3 promoter has multiple transcription factor binding sites (Figure 5A) and has not been previously investigated in CNS TB. MMP-3 promoter reporter analysis demonstrated that CoMTb increased the $2183 \mathrm{bp}$ fulllength promoter activity above CoMCont levels by 3.4fold at 24 hours (Figure 5B, $P<0.05$ ). A significant rise in promoter activity was seen with the 1612 bp promoter compared to the full length promoter in response to CoMTb stimulation $(P<0.01)$. Promoter activity was decreased compared to CoMCont levels when the shorter $642 \mathrm{bp}$ construct was stimulated with CoMTb, suggesting that this construct does not contain the minimal elements necessary to allow promoter activity in response to CoMTb.

\section{Discussion}

In this study, we have demonstrated that MMP-1 and MMP-3 secretion from monocyte-microglial networks are dependent on the p38, MAPK pathway in a cellular model of CNS TB. This control acts, at least in part, at the transcriptional level. We also defined that a key repressor element exists in the MMP-3 promoter upstream of -1612 from the transcriptional start site.

First, we delineated that CoMTb, but not CoMCont, drives dose-dependent MMP-1 and MMP-3 secretion. This effect peaked for MMP-3 at 72 hours, but MMP-1 secretion increased until 96 hours. Next, using phosphorarrays, we demonstrated that the ERK and AKT pathways were active in CoMCont-stimulated cells. These pathways are responsible for cell turnover, and therefore, basal expression in cell lines is commonly found [20]. Second, although there was increased phosphorylation of these pathways with CoMTb, the p38 pathway, and in particular the $\mathrm{p} 38 \alpha$ and $\gamma$ subunits, stood out as principle targets of CoMTb-activated signaling. This was confirmed in kinetic studies where biphasic phosphorylation of p38 was observed. Biphasic p38 activation is linked to the apoptotic pathway, which is activated only after the second peak in phosphorylation [21]. No specific subunit analysis was performed in our experiments, because the antibody used recognizes epitopes common to all four p38 subunits. However, M.tb has also been shown to directly drive p38 phosphorylation in microglia. Yang et al. demonstrated that sonicated (therefore dead) M.tb stimulated p38 phosphorylation in a toll-like receptor (TLR)2-dependent manner [22]. In our study, p38 $\alpha$ activation was most marked. Conditional knockout of p38 $\alpha$ in murine macrophages differentially affects bacterial phagocytosis and LPS-induced TNF- $\alpha$, but not IL- 6 , secretion via the attenuation of the transcription factor cAMP response element-binding protein (CREB) but not the NF-kB pathway [23]. There is emerging evidence that p38 inhibition may have a
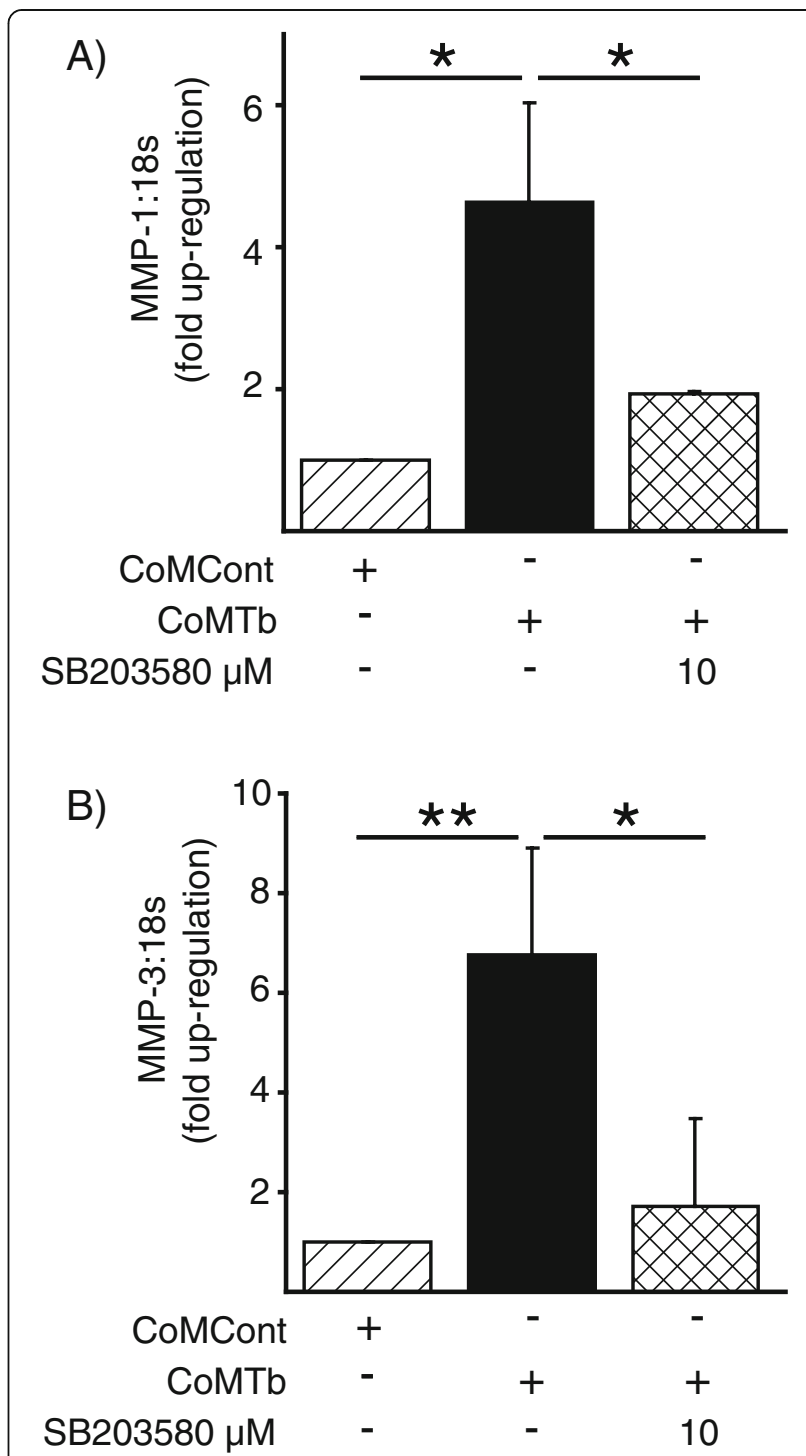

Figure 4 Matrix metalloproteinase (MMP)-1 and MMP-3 gene expression is suppressed by p38 inhibition. (A) Shows MMP-1 and (B) shows MMP-3 gene expression. mRNA from microglia stimulated for 24 hours with conditioned media from tuberculosis (TB)-infected monocytes (CoMTb) was analyzed by real-time PCR, normalized to $18 \mathrm{~S}$ RNA and expressed as fold-change relative to mRNA from 24 hour-conditioned media from control monocytes (CoMCont). The mean \pm SD from three experiments are shown. Data were analyzed by one-way analysis of variance, followed by Tukey's multiple comparison test. ${ }^{*} P<0.05,{ }^{* *} P<0.01$.

therapeutic role in the treatment of cerebral ischaemia, where it reduces neuronal death and microglial activation suggesting a possible translational application for our data [24]. In addition, we found that MMP-3 was solely p38dependent, and thus, p38 appears to be a central cellular control point for MMP-1 and MMP-3 secretion in microglial cells.

The high ERK basal phosphorylation up-regulated by CoMTb observed in the MAP kinase array was confirmed 


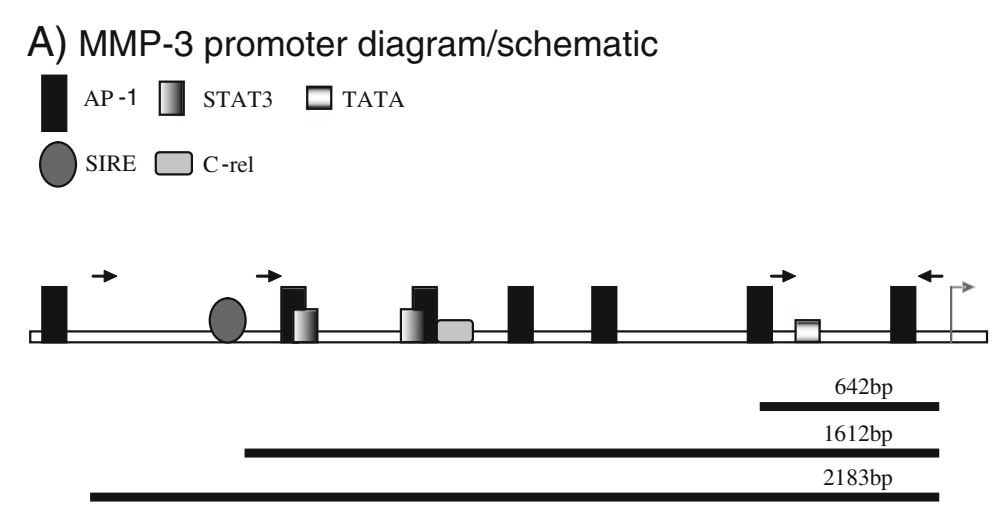

B) MMP-3 promoter reporter

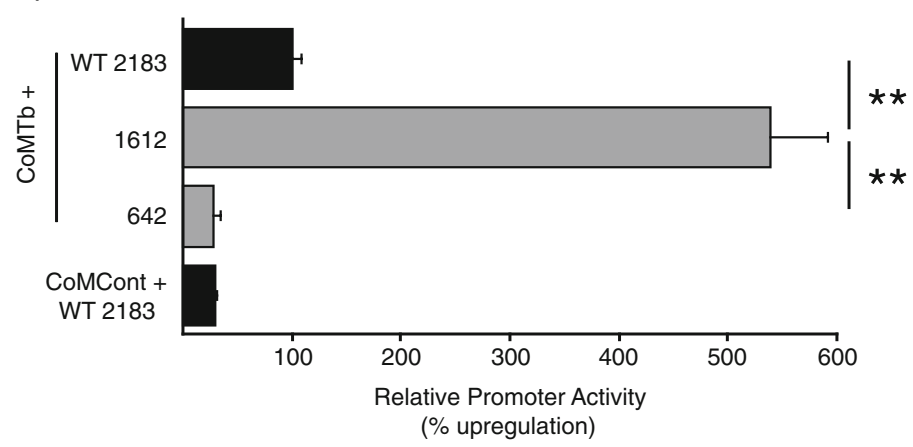

Figure 5 Matrix metalloproteinase (MMP)-3 promoter activity. (A) Schematic representation of full-length MMP-3 promoter demonstrating relevant promoter binding sites (shaded boxes). Right to left arrow represents transcription start site. Left to right arrows represent transcription stopping sites on the three promoter constructs, for which the length is indicated by the black line below. Of note, the 1612 bp construct disrupts a stromelysin IL-1 responsive element (SIRE) binding site. (B) MMP-3 promoter deletion constructs. Microglial cells were transiently transfected with full-length promoter (black bars) or deletion constructs (gray bars), and stimulated with, conditioned media from control monocytes (CoMCont) or conditioned media from tuberculosis (TB)-infected monocytes (CoMTb) for 24 hours. CoMTb-stimulated full-length MMP-3 promoter was designated as maximal luciferase activity (100\%), and other results are expressed as a percentage of this maximum. Bars are mean \pm SD from three samples and represent at least three separate experiments performed in triplicate. Data were analyzed by the Student $t$-test $\left({ }^{* *} \mathrm{P}<0.01\right)$.

by western blotting. However, since in cell lines, high basal ERK activity is driven by the immortalization process, we chose not to study this pathway further. However, ERK activation is known to contribute to the control of MMP secretion in the CNS, macrophages and other cells, suggesting a ubiquitous, rather than specific, cellular role for this MAPK $[16,25,26]$. Specifically, further experiments in primary cells are warranted to confirm these preliminary findings. Gene expression data exactly reflected secretion data although the extent of any post-transcriptional effect on MMP-1 regulated by p38 was not investigated in the present study.

Finally promoter-reporter studies demonstrated CoMTbspecific up-regulation of promoter activity, and also indicated that a repressor element exists in the upstream part of the promoter $(-1612$ to -2183$)$. The exact repressor element demonstrated to be acting here is as yet undefined. Tantalizingly the 1612 bp construct exactly disrupts a previously reported SIRE binding site, a transcription factor associated with down-regulation of MMP-3 secretion in fibroblasts [27]. In microglial cells, we have previously proven that the observed CoMTb effect involves synergy between TNF- $\alpha$ and IL-1 $\beta$ for MMP-3 but not MMP-1 secretion, supporting the role of this latter cytokine [9]. However, unlike in respiratory epithelial cells and macrophages, we have been unable to demonstrate an additional effect of $M$. $t b$-derived antigens on secretion of MMPs or p38 MAPK signalling via TLR2, respectively, in this model system $[14,15]$.

\section{Conclusions}

We have demonstrated that in CNS TB, MMP-1 and MMP-3 secretion from monocyte-microglial networks are critically dependent upon the p38 MAP kinase pathway, and this pathway may warrant further investigation as a therapeutic target in CNS TB.

\section{Abbreviations}

ANOVA: Analysis of variance; AP: Activator protein; bp: Base pairs; cfu: Colony forming units; CoMCont: Conditioned media from control monocytes; CoMTb: Conditioned media from tuberculosis-infected monocytes; CNS: Central 
nervous system; CREB: cAMP response element-binding protein CSF: Cerebrospinal fluid; Ct: Cycle threshold; DMEM: Dulbecco's modified Eagle's medium; ELISA: Enzyme-linked immunosorbent assay; ERK: Extracellular signalregulated mitogen-activated protein kinase; FCS: Fetal calf serum; HRP: Horseradish peroxidase; IL: Interleukin; MAPK: Mitogen-activated protein kinase; MMP: Matrix metalloproteinase; MSFM: Macrophage serum-free medium; NF-Kb: Nuclear factor-KB; OD: Optical density; qPCR: Quantitative polymerase chain reaction; SIRE: Stromelysin IL-1 responsive element; TB: Tuberculosis; TIMP: Tissue inhibitor of metalloproteinase; TLR: Toll-like receptor; TNF: Tumour necrosis factor.

\section{Competing interests}

The authors declare no conflict of interest.

\section{Authors' contributions}

JAG, LR, RM, PTE and JSF designed the experiments, which were undertaken by JAG, RM and SD. TP and LR designed, produced and purified the MMP-3 promoter. JAG wrote the first draft of the manuscript. JAG, LR, RM, PTG and JSF contributed to editing the manuscript. All authors read and approved the final manuscript.

\section{Acknowledgements}

JAG was supported by the Mason Medical Foundation and by a Medical Research Council (UK) Clinical Training Fellowship (G0500385). RM is supported by a Wellcome Trust Clinical Research Training Fellowship. SD was supported by a vacation grant from The Pathology Society, UK. JSF and PTE are grateful for support from the National Institute for Health Research (NIHR) Biomedical Research Centre funding scheme. JSF acknowledges the support of the Imperial College Wellcome Centre for Clinical Tropical Medicine. The funders had no role in study design, data collection and analysis, decision to publish, or preparation of the manuscript.

\section{Author details}

'Section of Infectious Diseases and Immunity and the Imperial College Wellcome Trust Centre for Clinical Tropical Medicine, Hammersmith Campus, Imperial College London, London W12 ONN, UK. ${ }^{2}$ Currently: Clinical and Experimental Sciences, University of Southampton, Southampton SO16 6YD, UK

Received: 28 March 2013 Accepted: 10 August 2013

Published: 26 August 2013

\section{References}

1. World Health Organization: Global Tuberculosis Control. Geneva; 2010

2. Elkington P, Shiomi T, Breen R, Nuttall RK, Ugarte-Gil CA, Walker NF, Saraiva L, Pedersen B, Mauri F, Lipman M, Edwards DR, Robertson BD, D'Armiento J, Friedland JS: MMP-1 drives immunopathology in human tuberculosis and transgenic mice. J Clin Invest 2011, 121:1827-1833.

3. Yong W, Power C, Forsyth P, Edwards DR: Metalloproteinases in biology and pathology of the nervous system. Nat Rev Neurosci 2001, 2:502-511.

4. Parks WC, Mecham RP: Matrix metalloproteinases. San Diego: Academic Press; 1998.

5. Nagase $H$, Visse $R$, Murphy $G$ : Structure and function of matrix metalloproteinases and TIMPs. Cardiovasc Res 2006, 69:562-573.

6. Agrawal S, Anderson P, Durbeej M, van Rooijen N, Ivars F, Opdenakker G, Sorokin LM: Dystroglycan is selectively cleaved at the parenchymal basement membrane at sites of leukocyte extravasation in experimental autoimmune encephalomyelitis. J Exp Med 2006, 203:1007-1019.

7. Prinz M, Mildner A: Microglia in the CNS: immigrants from another world. Glia 2011, 59:177-187.

8. Green JA, Dholakia S, Janczar K, Ong CW, Moores R, Fry J, Elkington PT, Roncaroli F, Friedland JS: Mycobacterium tuberculosis-infected human monocytes down-regulate microglial MMP-2 secretion in CNS tuberculosis via TNFalpha, NFkappaB, p38 and caspase 8 dependent pathways. J Neuroinflammation 2011, 8:46.

9. Green JA, Elkington PT, Pennington CJ, Roncaroli F, Dholakia S, Moores RC, Bullen A, Porter JC, Agranoff D, Edwards DR, Friedland JS: Mycobacterium tuberculosis upregulates microglial matrix metalloproteinase- 1 and -3 expression and secretion via NF-kappaB- and Activator Protein-1-dependent monocyte networks. J Immunol 2010, 184:6492-6503.
10. Nuttall RK, Silva C, Hader W, Bar-Or A, Patel KD, Edwards DR, Yong W: Metalloproteinases are enriched in microglia compared with leukocytes and they regulate cytokine levels in activated microglia. Glia 2007, 55:516-526.

11. Green JA, Tran CT, Farrar JJ, Nguyen MT, Nguyen PH, Dinh SX, Ho ND, Ly CV, Tran HT, Friedland JS, Thwaites GE: Dexamethasone, cerebrospinal fluid matrix metalloproteinase concentrations and clinical outcomes in tuberculous meningitis. PLoS One 2009, 4:e7277.

12. Harris JE, Nuttall RK, Elkington PT, Green JA, Horncastle DE, Graeber MB, Edwards DR, Friedland JS: Monocyte-astrocyte networks regulate matrix metalloproteinase gene expression and secretion in central nervous system tuberculosis in vitro and in vivo. J Immunol 2007, 178:1199-1207.

13. Thwaites GE, Nguyen DB, Nguyen HD, Hoang TQ, Do TT, Nguyen TC, Nguyen QH, Nguyen TT, Nguyen NH, Nguyen TN, Nguyen NL, Nguyen HD, Vu NT, Cao HH, Tran TH, Pham PM, Nguyen TD, Stepniewska K, White NJ, Tran TH, Farrar JJ: Dexamethasone for the treatment of tuberculous meningitis in adolescents and adults. N Engl J Med 2004, 351:1741-1751.

14. Rand L, Green JA, Saraiva L, Friedland JS TEP: Matrix metalloproteinase-1 is regulated in tuberculosis by a paraminosalicylic acid-sensitive cascade. $\mathrm{J}$ Immunol 2009, 182:5865-5872.

15. Elkington PT, Green JA, Emerson JE, Lopez-Pascua LD, Boyle JJ, O'Kane CM, Friedland JS: Synergistic up-regulation of epithelial cell matrix metalloproteinase-9 secretion in tuberculosis. Am J Respir Cell Mol Biol 2007, 37:431-437.

16. Harris JE, Green JA, Elkington PT, Friedland JS: Monocytes infected with Mycobacterium tuberculosis regulate MAP kinase-dependent astrocyte MMP-9 secretion. J Leukoc Biol 2006, 81:548-556.

17. Elkington PT, Emerson JE, Lopez-Pascua LD, O'Kane CM, Horncastle DE, Boyle JJ, Friedland JS: Mycobacterium tuberculosis up-regulates matrix metalloproteinase-1 secretion from human airway epithelial cells via a p38 MAPK switch. J Immunol 2005, 175:5333-5340.

18. Elkington PT, Green JA, Friedland JS: Filter sterilization of highly infectious samples to prevent false negative analysis of matrix metalloproteinase activity. J Immunol Methods 2006, 309:115-119.

19. Elkington PT, Nuttall RK, Boyle JJ, O'Kane CM, Horncastle DE, Edwards DR, Friedland JS: Mycobacterium tuberculosis but not vaccine BCG specifically up-regulates matrix metalloproteinase-1. Am J Respir Crit Care Med 2005, 172:1596-1604.

20. Tommasi S, Zheng A, Weninger A, Bates SE, Li XA, Wu X, Hollstein M, Besaratinia A: Mammalian cells acquire epigenetic hallmarks of human cancer during immortalization. Nucleic Acids Res 2013, 41:182-195.

21. Lee HE, Berkowitz P, Jolly PS, Diaz LA, Chua MP, Rubenstein DS: Biphasic activation of p38MAPK suggests that apoptosis is a downstream event in pemphigus acantholysis. J Biol Chem 2009, 284:12524-12532.

22. Yang CS, Shin DM, Lee HM, Son JW, Lee SJ, Akira S, Gougerot-Pocidalo MA El-Benna J, Ichijo H, Jo EK: ASK1-p38 MAPK-p47phox activation is essential for inflammatory responses during tuberculosis via TLR2-ROS signalling. Cell Microbiol 2008, 10:741-754

23. Kang YJ, Chen J, Otsuka M, Mols J, Ren S, Wang Y, Han J: Macrophage deletion of p38alpha partially impairs lipopolysaccharide-induced cellular activation. J Immunol 2008, 180:5075-5082.

24. Strassburger M, Braun H, Reymann KG: Anti-inflammatory treatment with the p38 mitogen-activated protein kinase inhibitor SB239063 is neuroprotective, decreases the number of activated microglia and facilitates neurogenesis in oxygen-glucose-deprived hippocampal slice cultures. Eur J Pharmacol 2008, 592:55-61.

25. Kim JY, Kim WJ, Kim H, Suk K, Lee WH: The Stimulation of CD147 induces MMP-9 expression through ERK and NF-kappaB in macrophages: implication for atherosclerosis. Immune Netw 2009, 9:90-97.

26. Park $S$, Jung $H H$, Park YH, Ahn JS, Im YH: ERK/MAPK pathways play critical roles in EGFR ligands-induced MMP1 expression. Biochem Biophys Res Commun 2011, 407:680-686.

27. Borghaei RC, Rawlings PL Jr, Javadi M, Woloshin J: NF-kappaB binds to a polymorphic repressor element in the MMP-3 promoter. Biochem Biophys Res Commun 2004, 316:182-188.

doi:10.1186/1742-2094-10-107

Cite this article as: Green et al:: In an in vitro model of human tuberculosis, monocyte-microglial networks regulate matrix metalloproteinase- 1 and -3 gene expression and secretion via a p38 mitogen activated protein kinase-dependent pathway. Journal of Neuroinflammation 2013 10:107. 